\title{
Autoimmunity in hereditary retinal degeneration. I. Basic studies
}

\author{
SUSAN M CHANT, JOHN HECKENLIVELY, AND ROBERTA H MEYERS-ELLIOTT \\ From the Jules Stein Eye Institute, Department of Ophthalmology, UCLA School of Medicine, Los Angeles
}

SUMMARY One hundred and sixteen patients with retinitis pigmentosa (RP), 64 patients with other eye diseases, and 36 control subjects with no known eye disease were examined for antiretinal autoimmune activity. Sera were screened by indirect immunofluorescence on normal donor human eye sections to detect antibodies to human retinal antigens. Forty three of $116 \mathrm{RP}$ patients (37\%), 21 out of 64 non-RP patients with other eye diseases (33\%), and 1 out of 42 controls ( $2 \%)$ had antibodies reacting with donor eye retinal antigens. Lymphocytes were tested by an in vitro transformation assay to detect cell mediated immunity to retinal antigens. Sixteen RP patients $(19 \%), 11$ non-RP patients $(18 \%)$, and four controls $(10 \%)$ showed lymphocyte sensitisation. Autoimmune responses were detected in many degenerative ocular disorders, but it is not known if they play a contributory pathogenic role.

Over the past 10 years various studies have examined the role of the immune system in retinitis pigmentosa (RP), a group of hereditary retinal degenerative disorders characterised by pigmentary retinopathy, progressive visual field loss, and nyctalopia. In 1973 Rahi reported that these patients had raised levels of serum IgM, though no specificity was assigned to the antibody.' Spiro et al. failed to confirm Rahi's findings but suggested that the discrepancy may have been from variations in methods, antisera, environmental influences, and composition of the RP groups used in the two studies. ${ }^{2}$ Spalton et al. using immunofluorescence techniques showed the binding of sera from RP patients to rat photoreceptor cells. ${ }^{3}$ Keltner et al. recently reported one case of photoreceptor degeneration in a cancer patient who had serum antibodies reactive with normal retinal tissue on the immunofluorescent complement fixation test. ${ }^{4}$

A number of studies have been made to look at cell mediated immunity in retinal degenerations. Lymphocytes from 17 out of 23 patients with retinal degenerative disease were cytotoxic to retinoblastoma cells in tissue culture, which indicates immune reactivity against retinal antigens by these patients. ${ }^{5}$ Retinoblastoma cells have previously been shown to contain some antigens present on normal photoreceptor cells. ${ }^{6}$ Rocha and Antunes showed cell

Correspondence to Roberta H Meycrs-Elliott, PhD, Jules Stein Eye Institute, UCLA School of Medicine, Los Angeles CA 90024, USA. mediated immunity in 34 out of $50 \mathrm{RP}$ patients with the use of the migration inhibition factor test.? Brinkman et al. using transformation and migration inhibition assays showed sensitisation to retinal antigens of lymphocytes from RP patients. ${ }^{8} \mathrm{~A}$ study by Heredia $e t$ al. showed sensitisation to retinal antigens in 26 out of 45 RP patients and presented evidence that lymphocytes from these patients also had a reduced response to phytohaemagglutinin in vitro and had diminished suppressor cell activity. ${ }^{9}$

While autoimmune activity has been well recorded in RP patients, it is not known whether it is specific to certain types of RP or how commonly it occurs in other types of retinal degeneration and ocular disease. To determine the extent and specificity of immune mechanisms active in a number of retinal degenerative disorders two tests of immune function were evaluated in blood samples from patients with RP, patients with non-RP ocular disease, and normal control subjects. The RP type was diagnosed as specifically as possible for each patient.

\section{Materials and methods}

Patients with progressive panretinal degenerations were evaluated with a comprehensive medical, ocular, and family history (pedigree analysis); ophthalmological examination; and tests consisting of Goldmann visual fields, electroretinogram, dark 
adaptation final rod threshold, fundus photography, and fluorescein angiography.

The patients with hereditary retinal degenerations were classified as precisely as possible by the above tests and examination results by means of previously published methods and rationale ${ }^{10-14}$ and placed in RP subgroups of: rod-cone and cone-rod degeneration by inheritance, choroideremia, preserved paraarteriole retinal pigment epithelium RP, pigmented paravenous retinochoroidal atrophy, and Usher syndrome.

Other retinal degenerations tested included congenital stationary nightblindness, Stickler's syndrome, luetic chorioretinitis, choroiditis, and sympathetic ophthalmia. These diseases were tested as an aid to understanding the specificity of the immunological responses. The control group consisted of volunteers with no known eye problems.

$10 \mathrm{ml}$ of peripheral venous blood was drawn into plain tubes for serum samples and $30 \mathrm{ml}$ into heparinised tubes for isolation of lymphocytes. Blood was drawn from $116 \mathrm{RP}$ patients, 64 non-RP patients with other eye disorders, and 42 normal volunteers without eye disorders who had given informed consent.

Isolation of serum and lymphocytes. Blood drawn for serum samples was allowed to clot and was centrifuged at $2000 \mathrm{rpm}$ for $10 \mathrm{~min}$. Then the serum was removed and stored at $-20^{\circ} \mathrm{C}$ until testing.

To isolate lymphocytes blood was centrifuged at $2000 \mathrm{rpm}$ for $10 \mathrm{~min}$. The buffy coat was removed, diluted with an equal volume of RPMI 1640 (Flow Laboratories, Inglewood, California), and layered on to $5 \mathrm{ml}$ cushions of Ficoll-paque (Pharmacia Fine Chemicals, Piscataway, New Jersey). After centrifugation at $2000 \mathrm{rpm}$ for $20 \mathrm{~min}$ the layer of cells at the interface was recovered, washed three times in RPMI 1640, and stored in freezing medium (RPMI $1640,10 \%$ DMSO, $10 \%$ glycerol, $10 \%$ sterile, heat inactivated human $\mathrm{AB}$ serum) at $-70^{\circ} \mathrm{C}$ until testing.

Immunofluorescence. Serum samples were tested by the method of Sainte-Marie ${ }^{15}$ to detect circulating antibodies to retinal antigens. A positive control consisted of human retinal sections incubated with rabbit antibody to human retina. A negative control consisted of human retinal sections incubated with saline in place of serum in the first step. Direct staining of sections with fluorescein conjugated goat antihuman IgG and IgM (Meloy, Springfield, Virginia 22151) was carried out to determine that there were no immunoglobulins bound to the retina prior to incubation with sera. Sera from patients and controls were examined for the presence of antibodies by means of fluorescein conjugated goat antihuman IgG and IgM.

In vitro transformation assays. Lymphocytes were tested by in vitro transformation assays with the use as antigens of crude human retinal extract $(250,100$, and $50 \mu \mathrm{g} / \mathrm{ml} ; 20 \mu \mathrm{l} / \mathrm{well}$ ), and a bovine rod outer segment preparation $(250,100$, and $50 \mu \mathrm{g} / \mathrm{ml} ; 20$ $\mu \mathrm{l} /$ well) ${ }^{16}$ Mitogens used to test functional viability of lymphocytes were concanavalin A (Miles-Yeda Ltd, Rehovot, Israel), $100 \mu \mathrm{g} / \mathrm{ml} ; 20 \mu \mathrm{l} / \mathrm{well}$, and phytohaemagglutinin (Difco Laboratories, Detroit, Michigan), $100 \mu \mathrm{g} / \mathrm{ml} ; 20 \mu \mathrm{l} /$ well. $(\mu \mathrm{g} / \mathrm{ml}=\mathrm{mg} / \mathrm{l}$.) The lymphocytes were incubated with the mitogens for three days or with antigens for six days at $37^{\circ} \mathrm{C}$ in a $5 \% \mathrm{CO}_{2}$ atmosphere. Tritiated thymidine (specific activity $25 \mathrm{mCi} / \mathrm{mmol}$ ) $10 \mu \mathrm{Ci} /$ well (Amersham, Arlington Heights, Illinois) was added four hours prior to harvesting. Results are expressed as a stimulation index (SI) calculated as:

$$
\mathrm{SI}=\frac{\text { counts per minute in presence of antigen }}{\text { counts per minute in absence of antigen }} \text {. }
$$

This ratio allows for comparison of results from one assay to another. A stimulated index $>2.0$ was considered an indication of cell mediated activity against retinal antigens. ${ }^{8}$

The results of the immunofluorescence and sensitisation tests were analysed statistically for the three groups (RP patients, patients with non-RP eye problems, controls with no known eye problems) with the use of the $\chi^{2}$ test and analysis of variance. Statistical review was done by Coralee Yale of the UCLA Department of Biostatistics.

\section{Results}

Table 1 summarises the results of indirect immunofluorescence testing of sera from patients and controls. Forty three of 116 RP patients $(37 \%)$ showed antibodies to retinal antigens. The antibodies were not restricted to one particular type of retinitis pigmentosa but rather were seen in all types examined so far. Twenty one of 64 patients with non-RP eye disorders $(33 \%)$ also developed antibodies to retinal antigens in their serum. One of the 42 normal controls $(2 \%)$ had circulating antiretinal antibodies. The intensity of staining varied from weak to strong, but even weakly stained sections could be distinguished readily from those in which staining was absent (Figs. 1-3). Staining was seen most frequently in the rod outer segments, though sera from several patients also reacted with cell membranes either in the outer nuclear layer or, more often, throughout the entire retina.

Transformation assays. Results of in vitro transformation assays (Table 1) indicated sensitisation to retinal antigens in 16 out of $86 \mathrm{RP}$ patients $(19 \%), 11$ 
out of 64 patients with other eye diseases (17\%), and 4 out of 42 controls $(10 \%)$. There was no difference in mitogen response between patients and controls.

Table 1 Patients with autoantibodies to retinal antigens and/or sensitisation to retinal antigens

\begin{tabular}{|c|c|c|c|c|}
\hline \multirow[t]{2}{*}{ Patient } & \multirow[t]{2}{*}{ Diagnosis } & \multicolumn{2}{|c|}{$\begin{array}{l}\text { Immuno- } \\
\text { fluorescence } \\
\text { staining }\end{array}$} & \multirow[t]{2}{*}{$\begin{array}{l}\text { Stimu- } \\
\text { lation } \\
\text { index }\end{array}$} \\
\hline & & $I g G$ & $\lg M$ & \\
\hline \multicolumn{5}{|c|}{ Retinitis pigmentosa $(R P)$ type* } \\
\hline 1 & $\begin{array}{l}\text { Preserved para-arteriolar retinal } \\
\text { pigment epithelium (PPRPE) }\end{array}$ & + & - & $1 \cdot 6$ \\
\hline 2 & PPRPE & - & + & 1.4 \\
\hline 3 & PPRPE & + & + & NT \\
\hline 4 & PPRPE & - & + & $1 \cdot 4$ \\
\hline 5 & Simplex rod-cone & + & + & $2 \cdot 7-$ \\
\hline 6 & Simplex rod-cone & - & - & $2 \cdot 2$ \\
\hline 7 & Simplex rod-cone & + & + & $2 \cdot 5$ \\
\hline 8 & Simplex rod-cone & - & - & $2 \cdot 3$ \\
\hline 9 & Simplex rod-cone & + & - & $2 \cdot 7$ \\
\hline 10 & Simplex rod-cone & + & - & 1.4 \\
\hline 11 & Simplex rod-cone & - & + & 1.7 \\
\hline 12 & Simplex rod-cone & + & + & NT \\
\hline 13 & Simplex rod-cone & + & + & $1 \cdot 1$ \\
\hline 14 & Simplex rod-cone & + & + & NT \\
\hline 15 & Simplex rod-cone & + & - & $2 \cdot 1$ \\
\hline 16 & Simplex rod-cone & - & - & $2 \cdot 1$ \\
\hline 17 & Simplex rod-cone & + & + & $0 \cdot 3$ \\
\hline 18 & Multiplex rod-cone & - & + & $1 \cdot 6$ \\
\hline 19 & Simplex cone-rod & - & + & $2 \cdot 5$ \\
\hline 20 & Simplex cone-rod & + & + & $2 \cdot 0$ \\
\hline 21 & Simplex cone-rod & - & - & $2 \cdot 8$ \\
\hline 22 & Simplex cone-rod & - & + & $2 \cdot 8$ \\
\hline 23 & Autosomal recessive rod-cone & + & + & $1 \cdot 1$ \\
\hline 24 & Autosomal recessive rod-cone & - & + & $1 \cdot 6$ \\
\hline 25 & Autosomal recessive rod-cone & + & + & $1 \cdot 6$ \\
\hline 26 & Autosomal recessive rod-cone & - & + & 1.4 \\
\hline 27 & Autosomal recessive rod-cone & - & - & $3 \cdot 4$ \\
\hline 28 & Autosomal recessive cone-rod & - & + & $2 \cdot 1$ \\
\hline 29 & Autosomal dominant rod-cone & + & + & $1 \cdot 7$ \\
\hline 30 & Autosomal dominant rod-cone & - & + & $1 \cdot 5$ \\
\hline 31 & Autosomal dominant rod-cone & + & + & $1 \cdot 7$ \\
\hline 32 & Autosomal dominant cone-rod & - & + & NT \\
\hline 33 & $X$-linked recessive rod-cone & - & + & $1 \cdot 5$ \\
\hline 34 & $\mathrm{X}$-linked recessive rod-cone & + & + & $1 \cdot 1$ \\
\hline 35 & $\mathrm{X}$-linked recessive rod-cone & - & - & $2 \cdot 3$ \\
\hline 36 & $X$-linked recessive rod-cone & - & + & $1 \cdot 5$ \\
\hline 37 & $X$-linked recessive rod-cone & - & + & $1 \cdot 5$ \\
\hline 38 & $X$-linked recessive cone-rod & + & - & $1 \cdot 1$ \\
\hline 39 & Choroideremia & + & - & $1 \cdot 1$ \\
\hline 40 & Choroideremia & + & - & $2 \cdot 4$ \\
\hline 41 & Choroideremia & + & - & NT \\
\hline 42 & Choroideremia & - & + & $1 \cdot 5$ \\
\hline 43 & Choroideremia & + & - & $2 \cdot 4$ \\
\hline 44 & Usher syndrome & + & - & $1 \cdot 1$ \\
\hline 45 & Usher syndrome & - & + & $1 \cdot 6$ \\
\hline 46 & Usher syndrome & - & - & $3 \cdot 8$ \\
\hline 47 & Usher syndrome & - & + & $1 \cdot 6$ \\
\hline 48 & Usher syndrome & - & + & $1 \cdot 5$ \\
\hline 49 & Simplex cone-rod & - & + & $1 \cdot 5$ \\
\hline 50 & Simplex cone-rod & - & + & $1 \cdot 2$ \\
\hline 51 & Leber's amaurosis & - & + & 1.4 \\
\hline 52 & Congenital RP with macular & + & + & NT \\
\hline
\end{tabular}

Nine of the RP patients showing sensitisation in the transformation assays also developed circulating antiretinal autoantibodies. Five of the non-RP

Table 1 Patients with autoantibodies to retinal antigens and/or sensitisation to retinal antigens-continued

\begin{tabular}{lll}
\hline Patient Diagnosis & $\begin{array}{l}\text { Immuno- } \\
\text { fluorescence } \\
\text { staining }\end{array}$ & $\begin{array}{l}\text { Stimu- } \\
\text { lation } \\
\text { index }\end{array}$ \\
& IgG IgM & \\
\hline
\end{tabular}

Patients with non-RP ocular disease $†$

1 Choroiditis $+\quad+\quad 1.4$

2 Stickler syndrome $+t_{+}+3$

3 Measles retinopathy $+\quad$ - 1.0

4 Sympathetic ophthalmia $+\quad$ - 1.0

5 Regional RPE/choroidal atrophy + - 3.4

6 Luetic chorioretinitis $+\quad+\quad 1.1$

Luetic chorioretinitis $\quad-\quad+\quad 1.4$

8 Congenital stationary night blindness

11 Macular hole $+\quad$ - 0.1

12 Macular degeneration $\quad$ - $\quad$ - 2.2

13 Macular degeneration $\quad+\quad+\quad 2 \cdot 0$

14 Cataract $+\quad+\quad 1.5$

15 Cataract $+\quad+\quad 2.3$

16 Cataract/retinal detachment $\quad$ + $\quad+\quad 1.2$

17 Glaucoma/macular degeneration - _ $\quad 2 \cdot 0$

18 High myopia $\quad$ - $\quad+\quad 1.1$

19 High myopia - $\quad$ - 3.8

20 Detached retina $\quad$ + $\quad 1.6$

21 Retinal break $\quad-\quad$ - 2.8

22 Toxoplasmosis $+\quad+\quad 1.5$

23 Toxoplasmosis $+\quad+\quad 1.3$

24 Fundus flavimaculatis $\quad-\quad$ - $\quad 2.5$

25 Diabetic retinopathy + $\quad$ - 1.3

26 Chorioretinitis + $\quad 0.7$

27 Uveitis $+\quad+\quad 1.1$

Normal

$1+-\quad+\quad 2 \cdot 1$

$2 \quad-\quad-\quad-3.0$

3 - $\quad-\quad 3.3$

$4 \quad-\quad-\quad 2 \cdot 2$

NT $=$ No cells available for testing.

*'There were 64 patients with negative indirect immunofluorescence and stimulation index $<2 \cdot 0$ with the following diagnoses: PPRPE (1), pigmented paravenous retinochoroidal atrophy (5), simplex rod-cone (15), multiplex rod-cone (1), simplex cone-rod (7), recessive rod-cone (9), dominant rod-cone (2), dominant cone-rod (1), X-linked rod-cone (7), choroideremia (4), Usher syndrome (8), congenital RP with macular colobomata (2), sector RP (1), AlbersSchonberg (1), RP/diabetes/renal failure (1).

†There were 37 patients with negative indirect immunofluorescence and stimulation index $<2.0$ with the following diagnoses: Congenital stationary night blindness (1), macular oedema (1), macular/retinal degeneration (9), cataract (2), retinal tear (1), fundus flavimaculatus (1), diabetic retinopathy (3), birdshot chorioretinitis (2), surface wrinkling retinopathy (1), dominant optic atrophy (1), vitreous degeneration (1), hydroxychloroquine toxicity (1), retinal vasculitis (4), carrier X-linked (1), chorioretinitis (3), panretinal degeneration (1), RPE degeneration (1), high myopia (1).

¥There were 38 normal volunteers with no indirect immunofluoresence or stimulation index $>2 \cdot 0$. 
patients and one control also showed both sensitisation and positive immunofluorescence. Results of both tests are summarised in Table 2 .
Antibodies reactive with retinal antigens were present in significantly more RP and non-RP patients $(p=0 \cdot 001)$ when each group was compared with the
Fig. 1 Indirect

immunofluorescence on normal human retina showing IgM autoantibodies in serum from patient with PPRPE. Heavy staining of rod outer segments and a generalised lighter staining of outer nuclear cell membranes (arrows) can be seen $(\times 235)$.
Fig. 2 Indirect

immunofluorescence on normal human retina showing $\operatorname{Ig} G$ autoantibodies in serum from patient with $X$-linked recessive cone-rod degeneration. Note strong staining (arrows) of cell membranes in outer nuclear layer $(\times 235)$.
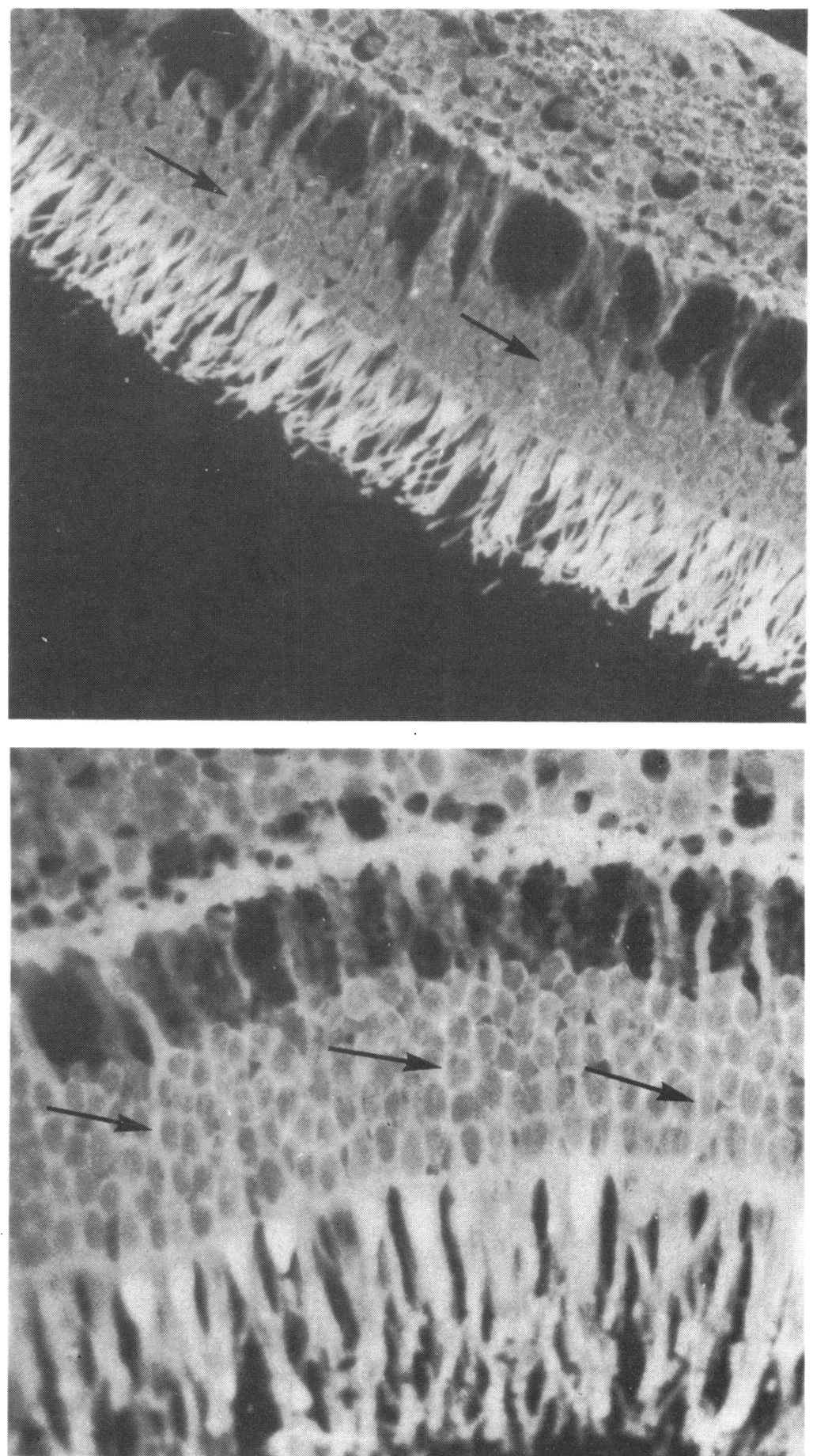


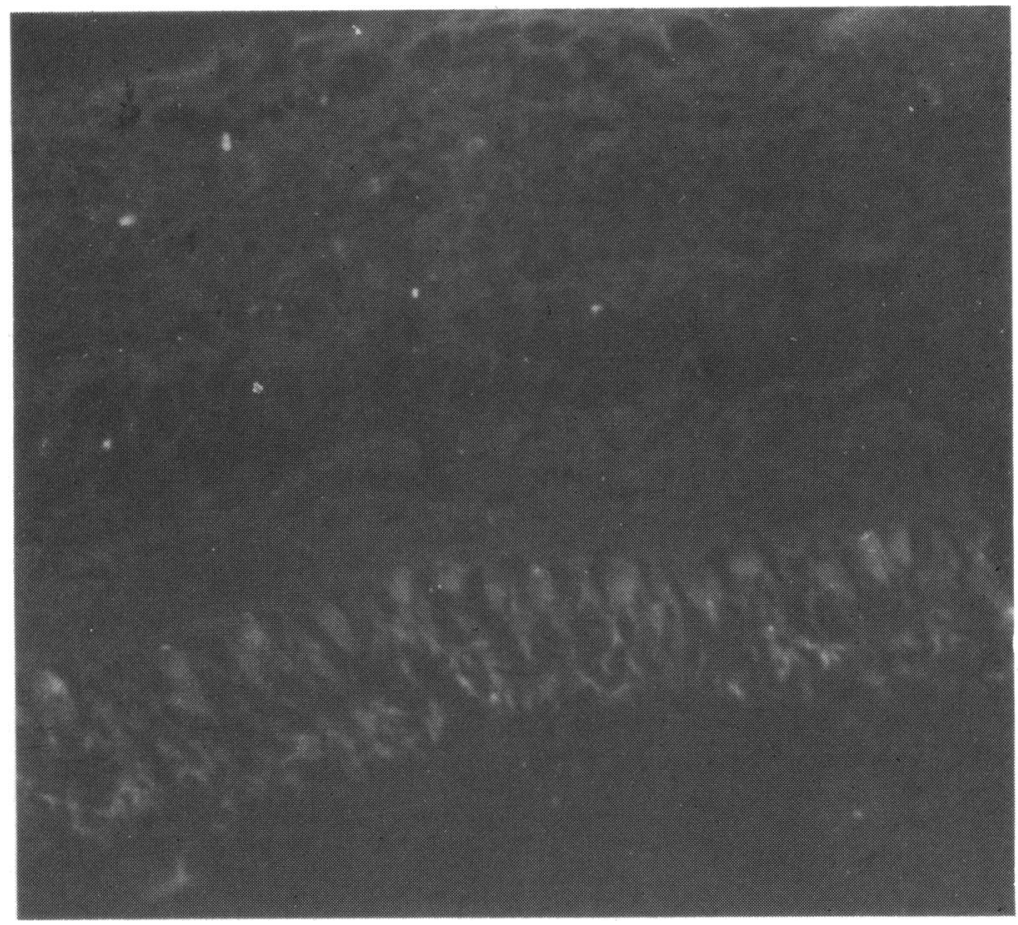

Fig. 3 Indirect immunofluorescence on normal human retina showing lack of staining with serum taken from normal control. Autofluorescence of the cones is evident in this section $(\times 235)$.

Table 2 Summary of immune responses to retinal antigens

\begin{tabular}{llll}
\hline & $\begin{array}{l}R P \\
\text { patients }^{*}\end{array}$ & $\begin{array}{l}\text { Non-RP } \\
\text { patients }\end{array}$ & $\begin{array}{l}\text { Normal } \\
\text { controls }\end{array}$ \\
\hline Autoantibodies & $43 / 116$ & $21 / 64$ & $1 / 42$ \\
& $(37 \%)$ & $(33 \%)$ & $(2 \%)$ \\
Sensitisation & $16 / 85$ & $11 / 64$ & $4 / 42$ \\
& $(19 \%)$ & $(18 \%)$ & $(10 \%)$ \\
Autoantibodics and sensitisation & $9 / 86$ & $5 / 64$ & $1 / 42$ \\
& $(10 \%)$ & $(8 \%)$ & $(2 \%)$ \\
\hline
\end{tabular}

associated with many diseases involving degenerative processes (for example, inflammatory bowel disease, multiple sclerosis, thyroiditis, rheumatoid arthritis, autoimmune haemolytic anaemia). Even though they may not be the primary cause of a disease, they can aggravate a degenerative process by immunopathological mechanisms thereby causing the normally protective role of the immune system to turn into a destructive one.

Recent studies in RP evaluating immune responses ${ }^{*} \mathrm{RP}=\mathrm{Retinitis} \mathrm{pigmentosa}$.
$\dagger$ Non- $\mathrm{RP}=$ Eye disease other than retinitis pigmentosa.

control group, but the incidence of antibodies to retinal antigens in the two patient groups did not differ significantly $(p=0.7622)$. The transformation assay, which indicates sensitisation of the cell mediated arm of the immune response, was not significantly different in any of the three groups: RP versus non-RP $p=0 \cdot 8668, R P$ versus normal $p=0.3963$, non-RP versus normal $p=0.4312$. Because of the small number of patients in each diagnostic category so far it was not possible to show significant differences between responses of patients with specific disease types with the use of either test of immunological function.

\section{Discussion}

Autoimmune mechanisms have been found to be to retinal antigens suggest the possibility that the immune system may contribute to the retinal degenerative process. ${ }^{1-3,5-9}$ Our results from the screening of sera to detect antibodies to retinal antigens indicate that autoimmune mechanisms are active in many patients with ocular degenerative disorders. A statistically significant $(p<0 \cdot 001)$ number of patients with both RP and non-RP eye disorders have circulating antibodies with activity directed towards retinal antigens as compared with normal controls. The transformation assays, which detect sensitised lymphocytes and indicate cellular rather than humoural mechanisms, showed no differences between patients and controls. If immune mechanisms do contribute to retinal ocular degeneration, it is more likely that they do so by the production of cytotoxic antibodies than by cell mediated mechanisms. 
Though an analysis of variance was done to evaluate the various ocular degenerative disorders, no pattern has emerged as yet with respect to differences in immune reactivity in the various types of degeneration. The autoimmune response appears to be an associated response with all types of ocular degeneration. We did note, however, several staining patterns on indirect immunofluorescence, in which specific parts of the retina are selectively involved (rod outer segments only, membranes only, or both membranes and rod outer segments). Further studies looking at differential staining patterns may eventually correlate with some disease types.

Patients with pigmented paravenous retinochoroidal atrophy (PPRCA) were grouped arbitrarily with the RP subgroups, since all these patients had a pigmentary retinopathy, and some of them showed a progressive retinal degeneration. However, it should be noted that many PPRCA patients have stationery disease, and the PPRCA is a degenerative pattern in which the common finding in all cases is retinal paravenous degenerative disease. ${ }^{17}$ Evaluation of the immune status of the PPRCA group was believed to be particularly pertinent, since the pathogenic mechanism for the paravenous degeneration is not understood.

Evidence of autoimmune reactivity in response to retinal antigens was found in 21 out of 64 non-RP patients and four control subjects in our studies. It is not surprising that such reactions are taking place in eye disorders other than RP, since immune reactions are seen frequently in eye disorders (for example, uveitis, sympathetic ophthalmia). The statistically significant difference between patients and controls showing antiretinal activity suggests that the immune reaction in these patients may be related to their retinal degeneration. The immune reactivity in normal controls and patients with non-retinal ocular problems could reflect a cross reactivity between antigens in the retina and other tissues.

Our preliminary studies thus provide evidence of autoimmune activity in RP of all types as well as other ocular degenerative disorders. Whether these mechanisms play a pathological role clinically has not yet been shown. If autoimmune mechanisms are shown to play a pathogenic role in the degenerative process, and if criteria can be established for therapeutic immunosuppression without endangering the health of the patient, it may be possible to slow the degener- ative process in selected patients who show strong autoimmune reactivity against retinal components.

This research was supported by a center grant from the National Retinitis Pigmentosa Foundation, a grant-in-aid from the National Society to Prevent Blindness (SMC), USPHS grants EY 1309 and EY 00331 from the National Eyc Institute, and a Rescarch To Prevent Blindness, Inc. rescarch manpower award (RME).

\section{References}

1 Rahi AHS. Autoimmunity and the retina. II. Raised serum IgM levels in retinitis pigmentosa. Br J Ophthalmol 1973; 57: 904-9.

2 Spiro R, Weleber R, Kimberling W. Serum IgM in retinitis pigmentosa-a genetic study. Clin Genet 1978; 13: 295-304.

3 Spalton DJ, Rahi AHS, Bird AC. Immunologic studies in retinitis pigmentosa associated with retinal vascular leakage. $\mathrm{Br} \mathrm{J}$ Ophthalmol 1978; 62: 183-7.

4 Keltner JL, Roth AM, Chang RS. Photoreceptor degeneration: Possible autoimmune disorder. Arch Ophthalmol 1983; 101: 564-9.

5 Char DH, Bergsma DR, Rabson AS, Albert DM, Herberman RB. Cell mediated immunity to retinal antigens in patients with pigmentary retinal degenerations. Invest Ophthalmol Visual Sci 1974; 13: 198-203.

6 Feldberg NT, Donoso LA. Surface and cytoplasmic antigens in retinoblastoma. Invest Ophthalmol Visual Sci 1980; 19: 1242-5

7 Rocha H, Antunes L. Primary retinitis pigmentosa-autoimmunity? Metabolic Ophthalmol 1977; 1: 153-6.

8 Brinkman CJJ, Pinckers AJLG, Broekhuyse RM. Immune reactivity to different retinal antigens in patients suffering from retinitis pigmentosa. Invest ophthalmol Visual Sci 1980; 19: 743-50.

9 Heredia CD, Vich JM, Huguet J, Garcia-Calderon JV, GarciaCalderon PA. Altered cellular immunity and suppressor cell activity in patients with primary retinitis pigmentosa. $\mathrm{Br} J$ Ophthalmol 1981; 65: 850-4.

10 Heckenlively JR. The classification of retinitis pigmentosa. Acta $X X I V$ Congress of ophthalmology. Philadelphia: Lippincott, $1983 ; 1: 34-7$.

11 Heckenlively JR. The hereditary retinal degenerations. In: Emory A, Rimoin D, eds. Principles and practice of medical genetics. Edinburgh: Churchill, 1983: 522-38.

12 Heckenlively JR, Martin DA, Rosales TO. Telangiectasia and optic atrophy on cone-rod degenerations. Arch Ophthalmol 1981; 99: 1983-91.

12 Heckenlively JR. Preserved periarteriole retinal pigment epithelium (PPRPE) in retinitis pigmentosa. $\mathrm{Br} \mathrm{J}$ Ophthalmol 1982; 66: 26-30.

14 Pearlman JT, Kamin DF, Kopelow SM, Saxton J. Pigmented paravenous retinochoroidal atrophy. Am J Ophthalmol 1975;80 630-5.

15 Sainte-Marie G. A paraffin embedding technique for studies employing immunofluorescence. J Histochem Cytochem 1962; 10: $250-6$.

16 Chant SM, Meyers-Elliott RH. Autoimmunity: A possible factor in the development of retinal degeneration in the RCS rat. Clin Imunol Immunopathol 1982; 22: 419-27.

17 Heckenlively JR, Kokame G. Pigmented paravenous retinochoroidal degeneration: Electrophysiological findings. Doc Ophthalmol Proc Ser in press. 